\title{
Expression and Characterization of Bluetongue Virus Serotype 21 VP7 Antigen: C- Terminal Truncated Protein has Significantly Reduced Antigenicity
}

\author{
Madhusudan HOSAMANI ${ }^{1)}$, Shinya SHIMIZU ${ }^{1)}$, Jiro HIROTA ${ }^{1)}$, Takehiro KOKUHO ${ }^{1)}$, Takayuki KUBOTA ${ }^{1)}$, \\ Satoko WATANABE ${ }^{1)}$, Masato OHTA $^{1)}$, Yoshihiro MUNETA ${ }^{1)}$ and Shigeki INUMARU ${ }^{1) *}$ \\ ${ }^{1)}$ National Institute of Animal Health, 3-1-5 Kan-non-dai, Tsukuba, Ibaraki 305-0856, Japan
}

(Received 20 May 2010/Accepted 8 December2010/Published online in J-STAGE 22 December 2010)

ABSTRACT. In the present study, group-specific antigen VP7 of bluetongue virus (BTV) serotype 21 isolated from cattle in Tochigi prefecture in Japan in 1994 was characterized by sequencing and expression. Gene was amplified from cDNA synthesized on viral dsRNA using reverse-transcriptase-PCR. Nucleotide sequence of this isolate showed high similarity with other published BTV VP7 sequences. Full-length and C-terminal truncated forms of VP7 were expressed in insect cells by a baculovirus gene expression system under control of the viral polyhedrin promoter. Expression of full-length recombinant VP7 was confirmed by immunoprecipitation with VP7 specific monoclonal antibody (8A3B.6, ATCC). Recombinant proteins expressed with or without 6x His-tag showed good expression levels in TN5 cells and reacted well with the monoclonal antibody in the indirect ELISA. However C-terminal truncated VP7 with His-tag failed to react with this monoclonal antibody, while poor antigenicity was evident when it was reacted with infected bovine serum. Reduced antigenicity of the latter suggested that C-terminal truncation affects 8A3B.6 epitope construction probably via inhibition of VP7 trimer structure formation.

KEY WORDS: baculovirus, bluetongue virus, serotype 21, VP7.

J. Vet. Med. Sci. 73(5): 609-613, 2011

Bluetongue(BT), an arthropod borne disease, which infects sheep, goat and cattle, has worldwide significance owing to its economic impact. The causative bluetongue virus (BTV) belongs to the orbivirus (Reoviridae), having 10 -segmented RNA genome. BT has been a global threat linked to livestock economy because of trade implications, although direct loss due to the disease is limited. Increased threat due to BT is evident with emergence of outbreaks involving new serotypes that were earlier not present in Europe. Wide spread outbreaks in Europe caused by BTV$8[5,9]$ have lead to introduction of the inactivated vaccine against this serotype recently. In Japan, first outbreak of BT in cattle and sheep occurred in 1994, and the subsequent outbreaks occurred only in 2001 [6] and 2005. However, BT has made a few incursions in the past as evidenced by seroepidemiology with mild and subclinical infection in cattle in certain parts of the country. Seroepidemiology conducted in 1974 has shown presence of BTV antibodies in domestic cattle in the absence of clinical symptoms, in Okinawa and Kyushu regions [14]. The epidemiology of the BT in Japan is however compounded by endemic Ibaraki virus infection in cattle making the serosurviellance a difficult task, because Ibaraki virus infected cattle sera show high cross-reaction quite high ratio on BT agar gel immunodiffusion test $[6,18]$. Presence of Ibaraki virus, a member of the epizootic hemorrhagic disease (EHD) group (Orbivirus), which is closely related to BTV is recorded in many parts of the country. Hence need for specific diagnostic

\footnotetext{
* Correspondence to: Dr Inumaru, S., Research Team for advanced Biologicals, National Institute of Animal Health, 3-15, Kan-non-dai, Tsukuba, Ibaraki 305-0856, Japan. e-mail: inumaru@affrc.go.jp
}

based on non-infectious diagnostic reagents for BTV is particularly relevant.

The major core protein, VP7 is known to be an important structural constituent of BTV, which forms the inner core with the underlying core protein VP3. VP7 is a major antigenic protein [19], and also a group-specific antigen, which is recommended by OIE to use for serological diagnosis of BTV in the form of competitive ELISA (cELISA) [16]. There is a potential practical application of BT diagnostic in Japan; hence we started to establish a diagnostic system using VP7 of Japanese strain BTV TO4-3 (serotype 21) isolated in 1994 as the antigen. Moreover, since BTV-21 VP7 gene has not been characterized, we sequenced the gene and produced the VP7 by gene expression in full length and truncated forms for evaluating their diagnostic potential.

\section{MATERIALS AND METHODS}

Cells: Hamster lung cell line (HmLu-1) was used for virus growth. Minimum essential medium Earle's salts [Invitrogen (Gibco), NY, U.S.A.] containing 5\% fetal bovine serum (FBS) was used for growth of cells, while the medium containing 1-2\% FBS was used for maintenance of cell line or for virus growth. For baculovirus expression, two insect cells viz. Spodoptera frugiperda (fall armyworm) derived cell line SF21AE and Trichoplusia ni (Cabbage looper) derived cell line BTI TN5B1-4 (TN5) were used, which were cultured with Sf900II medium [Invitrogen (gibco)] containing $10 \%$ of FBS and EX-CELL 405 medium (SAFC Bioscience, Kansas, U.S.A.), respectively. 
Virus: BTV serotype 21 (BTV TO4-3 HL19), which was earlier isolated from an outbreak of Japan in 1994 [6] and maintained at NIAH Japan was used. Virus was grown by infecting HmLu-1 cell monolayer under incubation at $37^{\circ} \mathrm{C}$. Infected cell suspension was harvested after appearance of more than $80 \%$ cytopathicity on day 4-5 and cell pellet obtained by low speed centrifugation was used for viral RNA isolation.

Isolation of RNA and cDNA synthesis: Viral RNA was extracted by treatment of infected cell pellet with TRIZOL reagent (Invitrogen, California, U.S.A.) for isolation of total RNA followed by precipitation of viral dsRNA using $\mathrm{LiCl}_{2}$ method. Gel purified S7 segment of viral RNA was reverse transcribed by MMLV-RT using gene-specific primers by employing ThermoScript MMLV-RT-PCR kit (Invitrogen) as per manufacturer's instructions except using dimethyl formamide at a final concentration of $5 \%(\mathrm{v} / \mathrm{v})$ in the reaction mixture. For amplification of the full length VP7 gene, primers were designed based on the consensus sequence generated by alignment of available gene sequences from the public database. The sequence of the primers VP7F1: 5, TGGGATCCATGGACACTATCGCWGCAAGAGC 3' and VP7R1: 5' TGGGATCCAGTGGRCTAYRCRTARGC GGCG 3'. Primers were flanked with BamHI site (underlined bases) to allow subcloning.

Cloning and sequencing of VP7 gene: PCR amplicon was purified and cloned into PCR-II blunt TOPO vector (Invitrogen). Three proper recombinant plasmids identified by restriction enzyme analysis were sequenced in an automated DNA sequencer (ABI 3100, Applied Biosystems, U.S.A.).

Generation of different constructs and production of recombinant viruses: Amplicons generated by PCR using different primers (Table 1) were cloned into transfer vector pACYM1 to produce recombinant viruses by homologous recombination with baculovirus genome. Plasmids containing full-length VP7 genes coding 349 amino acids without or with N-terminal His tag and truncated VP7 containing two-thirds of amino-terminal portion spanning 243 amino acids with His-tag on C-terminal (pACYM1/BTV21VP7, pACYM1/BTV21VP7-NH and pACYM1/BTV21-TrVP7$\mathrm{CH}$, respectively) were made and sequenced to verify correct orientation of the gene insert.

The LacZ gene recombinant Autographa californica nucleopolyhedrovirus (AcLacZ) [8] was used to construct recombinant baculovirus by homologous recombination. The plasmid constructs were co-transfected with AcLacZ viral DNA into SF21AE cells using cellfectin (Invitrogen) reagent. Transfectant supernatant was subjected to plaque purification and recombinant viruses were identified by PCR [12] using insert specific primer (VP7R4, Table 1) and flanking polyhedrin sequence. The recombinant virus clones were named Ac/VP7, Ac/VP7NH and Ac/TrVP7CH respectively. These clones were subjected to two rounds of plaque purification and amplified to high titres and used for expression analysis.

VP7gene expression: The recombinant virus clones were inoculated to BTI TN 5B1-4 (TN5) cells at MOI of 2 to 10. The expressed products were recovered from the infected cells after 3 to 4 days after inoculation.

$S D S-P A G E$ and Western blot: Expression of the protein in the TN5 cell was examined by SDS-PAGE. Infected cell lysates prepared in 2x SDS sample buffer were resolved in precast gels of $4-12 \%$ bis-tris acrylamide gel (Invitrogen) along with protein standards prestained marker (Biorad, California U.S.A.). Gels were stained with rapid stain CBB kit (Nacalai tesque, Kyoto Japan). For western blotting, proteins separated on PAGE were transferred onto polyvinylidene difluoride membranes. After blocking free sites with Tris buffered saline $(0.05 \mathrm{M}$ Tris, $0.138 \mathrm{M} \mathrm{NaCl}$, $0.0027 \mathrm{M} \mathrm{KCl}, \mathrm{pH} 8.0$ ) containing 3\% non-fat milk (Sigma, St. Louis U.S.A.), antigen was probed with both BTVinfected serum or VP7 specific monoclonal antibody (mAb) viz. 8A3B.6 (reference antibody developed against BTV17, CRL-1875 from ATCC, U.S.A.), following a standard procedure.

Immunoprecipitation: Reactivity of the recombinant antigen was examined by immunoprecipitation with $\mathrm{mAb}$ 8A3B.6. The test was performed using Seize X protein G immunoprecipitation kit (Pierce, Illinois U.S.A.) as described by the manufacturer. Purified viral antigen prepared from BTV infected HmLu-1 cell culture fluid was also used for binding to the mAb along with non-recombinant virus infected TN5 cell lysate as negative control. After washings, bound antigen was eluted into sample buffer and separated into the SDS-PAGE.

Purification of protein and protein assay: Purification of

Table 1. List of primers used for preparation of different constructs

\begin{tabular}{l}
\hline Primers used \\
\hline VP7F1:TGGGATCCATGGACACTATCGCWGCAAGAGC \\
VP7His-NF:TGGGATCCATG CATCACCATCACCATCACGACACTATCGCAGCAAGAGC \\
VP7His-TCR:TGGGATC $\boldsymbol{C}$ TA $\overline{\text { GTGATGGTGATGGTGATGTACCATTGCATTCTGCTGTGTA }}$ \\
VP7R1: TGGGATCCAGTGGRCTAYRCRTARGCGGCG \\
VP7R4: GAGCTAAAGACGTCGGGCGC \\
\hline Constructs expressed in insect cells \\
\hline VP7: VP7F1/VP7R1 1072bp (349aa) \\
VP7-NH: VP7His-NF/VP7R1 1090bp (349aa+6 His) \\
TrVP7-CH: VP7F1/VP7His-TCR 765 bp (243aa+6 His)
\end{tabular}


the recombinant VP7 with His tag was carried out using Probond purification system (Invitrogen) as per manufacturer's protocol. Protein concentration was estimated using densitometry with known concentrations of bovine serum albumin as the standard.

Indirect ELISA: Various dilutions of the purified antigen were coated onto the 96 well ELISA plate (Nunc, Roskilde Denmark) by incubation at $37^{\circ} \mathrm{C}$ for $1 \mathrm{hr}$ and unbound sites were blocked using blocking solution. Optimized dilutions of the mAb (8A3B.6) or infected bovine serum were used as primary antibody. After washing, horse radish peroxidase conjugated secondary antibody specific to either mouse or bovine immnoglobulins (Zymed Laboratories, San Francisco, U.S.A.) were added respectively and incubated further. After washing, color was developed using ABTS [2,2'-Azinoo-bis (3-ethylbenzothiazoline-6-sulfonic acid)] substrate and optical density (OD) was measured in ELISA reader at $405 \mathrm{~nm}$ (Tecan SPECTRAFluor Plus, Austria). Reactivity of truncated protein was compared with that of full-length protein. Truncated protein did not show reactivity with the mAb $8 \mathrm{~A} 3 \mathrm{~B} .6$, hence BTV serotype 21 infected bovine serum was used to check its antigenicity.

\section{RESULTS}

PCR amplification and sequencing of full-length VP7: Full-length VP7 gene of the expected size (1072 bp) was generated by PCR on the cDNA synthesized using the gene specific primers. VP7 sequence of this isolate (BTV T04-3) (submitted to DDBJ, Accession AB473807) revealed 90 to $97 \%$ of nucleic acid sequence identity and 82 to $99 \%$ of amino acid sequence identity with VP7 sequences of representative BTV serotypes available in the database.

Expression of recombinant protein: Recombinant products were accumulated in the recombinant virus infected TN5 sells. Expression of 36.5 and $37 \mathrm{kDa}$ proteins of VP7 and VP7-NH respectively were observed in Ac/VP7 and Ac/ VP7-NH infected cells and about $26 \mathrm{kDa}$ protein (TrVP7$\mathrm{CH}$ ) was in the Ac/TrVP7CH infected cells (Fig. 1). No expressed protein was detectable in the supernatants of the above constructs. Western blot using both VP7 specific monoclonal and polyclonal antibody from BTV-21-infected cow did not show any result with the expressed protein.

Immunoprecipitation: Crude lysates of the cells infected with recombinant clones were bound to mAb 8A3B. 6 immobilized on sepharose beads. Single band of 36.5 and $37 \mathrm{kDa}$ was observed in full-length constructs while no precipitated protein was observed in the truncated construct and non-recombinant virus (Fig. 2).

Purification of VP7-NH: His-tagged VP7 was purified using nickel chelating column chromatography and the yield of the purified protein was estimated to be $1.87 \mathrm{mg}$ per $7 \times 10^{6}$ TN5 cells in 4-days stationary culture. Protein was purified to near homogeneity as checked by SDS-PAGE (Fig. 2).

Indirect ELISA: The test was carried out using both $\mathrm{mAb}$ 8A3B.6 and the bovine polyclonal antiserum from the BTV-

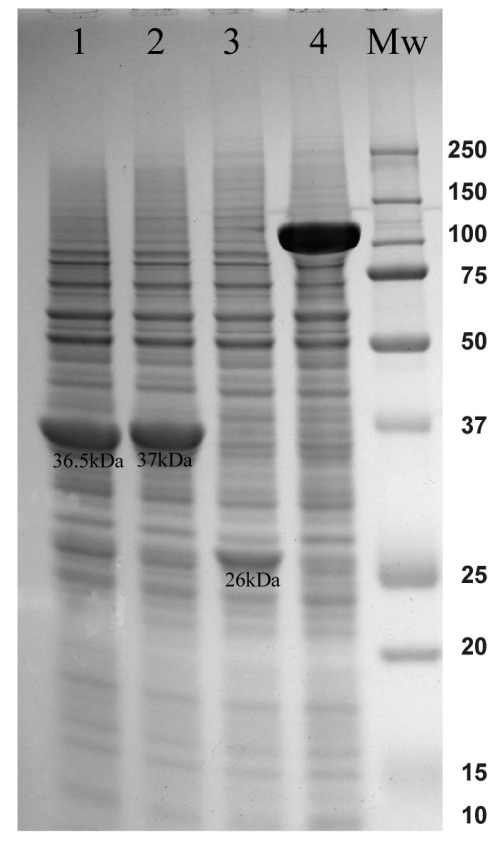

Fig. 1. SDS-PAGE analysis of VP7 expressed in TN5 cells using whole cell lysate. Expression of fulllength VP7 (Lane 1) using Ac/VP7 construct. Lane 2: Ac/VP7-NF with N-terminal His-tag, Lane 3: Ac/ VP7-truncated protein of $26 \mathrm{kDa}$, Lane 4: AcLacZ showing expression of LacZ gene, Lane Mw: Protein standard molecular weight markers (Biorad precision plus, dual colored)

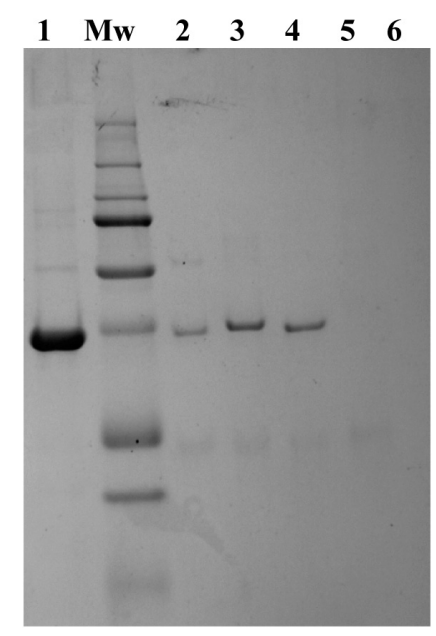

Fig. 2. Immunoprecipitation of the VP7 proteins using immobilized mAb. Lane 2-4: VP7, VP7-NH and Viral antigen (as positive control), respectively. Lysate from recombinant virus expressing truncated protein (TrVP7-CH) (lane 5) and the non-recombinant virus (negative control) processed similar to the above constructs (lane 6) did not show protein band. Purified VP7-NH using Ni-NTA chromatography is shown in lane 1 , next to the standard molecular weight marker (Mw) (Biorad). 


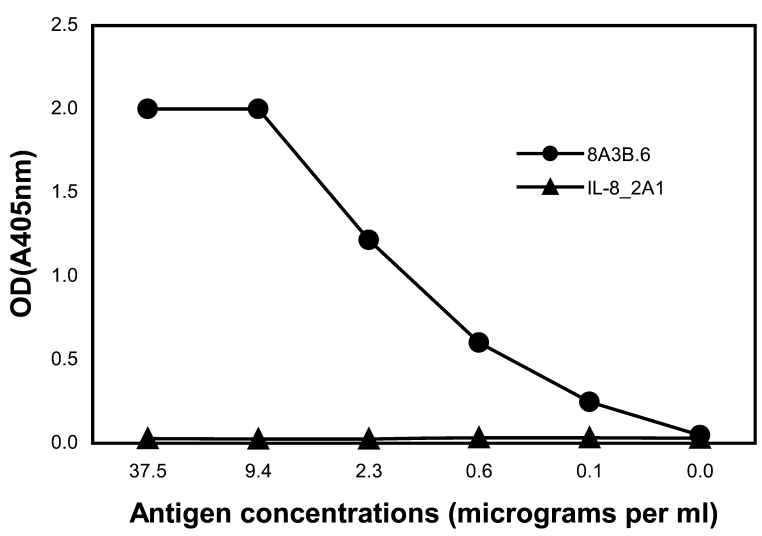

Fig. 3. Reactivity of the recombinant BTV-21 VP7-NH antigen in the ELISA, with monoclonal antibody 8A3B.6 (ATCC) specific to VP7. Purified VP7-NH antigen at various concentrations reacted with this $\mathrm{mAb}$ but not with the unrelated $\mathrm{mAb}$ (IL8_2A1) which served as negative antibody control in the assay.

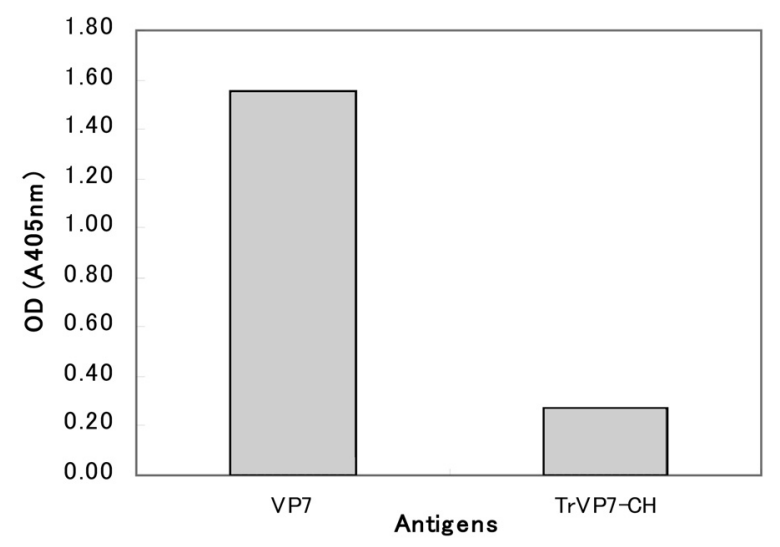

Fig. 4. Poor antigenicity of the truncated protein (TrVP7-CH) with infected bovine serum as compared to the full-length protein (VP7) in the ELISA.

21 experimentally infected cow. Full-length proteins with or without N-terminal His-tag showed comparable results against both $\mathrm{mAb}$ and the polyclonal antibody from BTV21-infected cow. Purified VP7-NH showed varying reactivity at fixed dilution of $\mathrm{mAb}$ in a dose dependent manner (Fig. 3). However truncated protein failed to react with the $\mathrm{mAb}$ while it showed poor antigenicity in comparison to the full- length recombinant VP7 (Fig. 4) with polyclonal antibody from BTV infected cattle.

\section{DISCUSSION}

Need for a reliable assay that can differentiate BTV infection from other related orbivirus infections such as Ibaraki disease in Japan has been substantiated. Use of recombinant antigen precludes handling of infectious virus and also over- comes the background reactivity resulting from use of whole virus antigen in the ELISA. Group-specific antigen expressed by various laboratories in baculovirus, E. coli, yeast or vaccinia virus $[3,10,13,15,17]$, has been widely employed in BT diagnostics.

In the present study, BTV-21 group-specific protein (VP7) has been sequenced and expressed in insect cells in three forms for their diagnostic application. Expression of full-length VP7, VP7-NH and truncated protein of expected molecular size was achieved as revealed by SDS-PAGE. In western blot using VP7 specific $\mathrm{mAb}$ and antibody from BTV-21-infected calf, these antigens failed to show signals under denaturing conditions. Inconsistent reactivity of VP7 to monoclonal or polyclonal antibodies in western blotting due to possible alteration in the epitope structure under denaturing conditions has been reported [21]. To overcome this, immunoprecipitation was used in the present study, where in VP7 and VP7-NH reacted with the immobilized $\mathrm{mAb}$ under native conditions, confirming the identity of the expressed protein.

Antigenicity of the recombinant products was further confirmed by ELISA using VP7 specific mAb. VP7 and VP7-NH appeared to show good antigenicity in the ELISA with no significant difference. These results are consistent with previous observations that presence of His-tag on the $\mathrm{N}$-terminal does not significantly affect the antigenicity of VP7 of Epizootic hemorrhagic disease virus [11], also an orbivirus, closely related to BTV. Addition of extra residue to N-terminal of VP7 will not affect the formation of viral cores of BTV [2].

Previous reports have shown that N-terminal of the VP7 has dominant epitope exposed on the surface [4]. This has been confirmed from later studies by Wang et al. [20] using $\mathrm{mAb} 20 \mathrm{E} 9$, that a conformation dependant epitope between 30 48aa in the VP7 polypeptide is surface accessible and is immunodominant. They also demonstrated that $\mathrm{mAb}$ 8A3B.6 competed with 20E9 for binding to viral antigen and reacted not with a monomeric but a putative assembled VP7. Since natural VP7 configurates trimer, it is indicating that the mAb 8A3B.6 recognizes an epitope located at least adjacent to that of $\mathrm{mAb} 20 \mathrm{E} 9$ epitope at the trimeric VP7 Nterminal region. Recombinant proteins produced by baculovirus gene expression systems are generally assembled as the same way as the natural proteins. The fact that recombinant VP7 expressed in this study was reactive with $\mathrm{mAb}$ 8A3B.6, suggested that the expressed VP7 formed trimers. Hence we also attempted to over express the recombinant antigen containing only two-third portion of VP7 (1-243aa) on N-terminal, with histidine tag on C-terminal. Truncated antigen however failed to react with mAb 8A3B.6 in immunoprecipitation and in ELISA. More over, when reacted with polyclonal antibody from BTV-21-infected bovine, it (TrVP7-CH) showed poor antigenicity in comparison to full-length proteins with or without His-tags (VP7 or VP7$\mathrm{NH}$ ) in ELISA.

These observations can be explained on the basis of crystallographic studies revealed for BTV-VP7 protein [7]. It is 
shown that there are 5 helices in the $\mathrm{N}$-terminal region and 4 helices in the $\mathrm{C}$-terminal region. Among them, two helices each in the N-terminal and C-terminal are located parallel. This structure seemed to provide rigid domain configuration. Therefore, it was presumed that elimination of Cterminal region possibly affected the accurate structure of the N-terminal region. It has been demonstrated that $\mathrm{N}$-terminal portion of the molecule is intimately involved in the formation of the VP7 trimers [20]. Therefore, the Cterminal truncation presumably affects the rigidity of VP7, thereby leading to improper assembly of VP7. On the other hand, there is another possibility that C-terminal histidine tag may affect the configuration. However, it may not be the case, since histidine tag is relatively short sequence and located distant to the N-terminal in the structure by SWISSMODEL prediction. Our data described in this report suggest that C-terminal portion of the VP7 is also closely involved in the formation of the trimers.

ACKNOWLEDGMENTS. This work was supported from Department of Biotechnology, GOI, New Delhi, under the associateship program 2006-07 and Ministry of Agriculture, forestry and Fisheries, Japan under the crisismanagement system consolidation project 2007-08. Technical staff of NIAH is gratefully acknowledged for their assistance.

\section{REFERENCES}

1. Appleton, J. A. and Letchworth, G. J. 1983. Monoclonal antibody analysis of serotype-restricted and unrestricted bluetongue viral antigenic determinants. Virology 124: 286-299.

2. Blois, H. L. and Roy, P. 1993. A single point mutation in the VP7 major core protein of bluetongue virus prevents the formation of core-like particles. J. Virol. 67: 353-359.

3. Cloete, M., Plessis, D. H., Dijk, A. A., Huismans, H. and Viljoen, G. J. 1994. Vaccinia virus expression of the VP7 of BTV-4 and its use as antigen in a capture ELISA. Arch. Virol. 135: 405-418.

4. Eaton, B. T., Gould, A. R., Hyatt, A. D., Coupar, B. E. H., Martyn, J. C. and White, J. R. 1991. A bluetongue sero-group reacting epitope in the amino terminal half of the major core protein VP7 is accessible on the surface of bluetongue virus particles. Virology 180: 687-696.

5. Enserink, M. 2006. Emerging infectious diseases. During a hot summer, bluetongue virus invades northern Europe. Science 313: $1218-1219$.

6. Goto, Y., Yamaguchi, O. and Kubo, M. 2004. Epidemiological observations on bluetongue in sheep and cattle in Japan. Vet. Ital. 40: 78-82.

7. Grimes, J., Basak, A. K., Roy, P. and Stuart, D. 1995. The crys- tal structure of bluetongue virus VP7. Nature 373: 167-170.

8. Kitts P. A., Ayres M. D. and Possee R. D. 1990. Linearization of baculovirus DNA enhances the recovery of recombinant virus expression vectors. Nucleic. Acids Res. 18: 5667-5672.

9. Landeg, F. 2007. Bluetongue outbreak in the UK. Vet. Rec. 161: 534-535.

10. Luo, L. and Sabara, M. I. 2008. Production, characterization and assay application of a purified, baculovirus-expressed, serogroup specific bluetongue virus antigen. Trans. Emerg. Dis. 55, 175-182.

11. Luo, L. and Sabara, M. I. 2005. Production of a recombinant major inner capsid protein for serological detection of epizootic hemorrhagic disease virus. Clin. Diagn. Lab. Immunol. 12: 904-909.

12. Malitschek, B. and Schartl, M. 1991. Rapid identification of recombinant baculoviruses using PCR. Biotechniques 11: 177178.

13. Martyn, J. C., Gould, A. R. and Eaton, B. T. 1990. High-level expression of the VP7 and the non-structural protein NS3 of BTV by yeast expression vector: Use of expressed VP7 as a group reactive antigen in blocking ELISA. Virus Res. 18: 67178.

14. Miura Y., Inaba Y., Tsuda T., Tokuhisa S., Sato K. and Akashi H. 1982. Seroepizootiological survey on bluetongue virus infection in cattle in Japan. Natl. Inst. Anim. Hlth. Q. 22: 154 158.

15. Oldfield, S., Adachi, A., Urakawa, T., Hirasawa, T. and Roy, P. 1990. Purification and characterization of the major groupspecific core antigens VP7 of bluetongue virus synthesized by a recombinant baculovirus. J. Gen. Virol. 71: 2649-2656.

16. OIE, Manual of Diagnostic Tests and Vaccines for Terrestrial Animals 2008 Chapter 2.1.3. BLUETONGUE 158-174.

17. Pathak, K. B., Biswas, S. K., Tembhurne, P. A., Hosamani, M., Bhanuprakash, V., Prasad, G., Singh, R. K., Rasool, T. J. and Mondal, B. 2008. Prokaryotic expression of truncated VP7 of bluetongue virus (BTV) and reactivity of the purified recombinant protein with all BTV type-specific sera. J. Virol. Methods. 152: $6-12$.

18. Shimizu, S., Toyota, I., Arishima, T. and Goto, Y. 2004. Frequency of serological cross-reactions between Ibaraki and bluetongue viruses using the agar gel immunodiffusion test. Vet. Ital. 40: $583-586$.

19. Wang, L. F., Hyatt, A.D., Whiteley, P. L., Andrew, M., Li, J. K. and Eaton, B. T. 1996. Topography and immunogenicity of bluetongue virus VP7 epitopes. Arch. Virol. 141: 111-123.

20. Wang, L. F., Scanlon, D. B., Kattenbelt, J. A., Mecham, J. O. and Eaton, B. T. 1994. Fine mapping of a surface-accessible, immunodominant site on the bluetongue virus major core protein VP7. Virology 204: 811-814.

21. Zhou, E.M. and Chan, M.K.H. 1996. Immunoblot assay as a confirmatory test of competitive ELISA for serodiagnosis of bluetongue. J. Vet. Diagn. Invest. 8: 365-367. 